\title{
Solvability of some boundary value problems involving $p$-Laplacian and non-autonomous differential operators
}

\section{Milena Petrini ${ }^{* *}$ (1)}

\author{
"Correspondence: \\ m.petrini@univpm.it \\ 'Dipartimento di Scienze \\ Matematiche, Università Politecnica \\ delle Marche, Ancona, Italy
}

\begin{abstract}
The paper deals with the existence and non-existence of solutions of the following nonlinear non-autonomous boundary value problem governed by the $p$-Laplacian operator:

$$
(P) \quad\left\{\begin{array}{l}
\left(h(t, x(t))\left|x^{\prime}(t)\right|^{p-2} x^{\prime}(t)\right)^{\prime}=g\left(t, x(t), x^{\prime}(t)\right) \quad \text { a.e. } t \in \mathbb{R} \\
x(-\infty)=a, \quad x(+\infty)=b
\end{array}\right.
$$

with $a<b$, where $a$ is a positive, continuous function and $g$ is a Caratheódory nonlinear function.

We prove an existence result, underlying the relationship between the behavior of $g(t, x, \cdot)$ as $y \rightarrow 0$ related to that of $g(\cdot, x, y)$ and $h(\cdot, x)$ as $|t| \rightarrow+\infty$.

MSC: Primary 34B40; 34C37; secondary 34B15; 34L30

Keywords: Boundary value problems; Unbounded domains; Heteroclinic solutions; Nonlinear differential operators; $p$-Laplacian operator; $\Phi$-Laplacian operator
\end{abstract}

\section{Introduction}

Differential equations involving the $p$-Laplacian operator and its generalization, the socalled $\Phi$-Laplacian, have been widely studied due to several applications in various sciences. Indeed, many models in non-Newtonian fluid theory, diffusion of flows in porous media, nonlinear elasticity, and theory of capillary surfaces can be expressed in terms of such differential operators.

The simplest form of a differential equation involving the $\Phi$-Laplacian operator is

$$
\left(\Phi\left(x^{\prime}\right)\right)^{\prime}=g\left(t, x, x^{\prime}\right)
$$

and it has been studied in many papers [1, 2, 7, 8, 12, 14-16, 23]. We also quote [19] for systems of differential equations, [13] for differential inclusions, [17, 18, 24] for systems of differential inclusions.

More recently, other types of differential operators, governed by an increasing function $\Phi$, possibly singular and not necessarily surjective, have been considered. The theory on

(c) The Author(s) 2019. This article is distributed under the terms of the Creative Commons Attribution 4.0 International License (http://creativecommons.org/licenses/by/4.0/, which permits unrestricted use, distribution, and reproduction in any medium, provided you give appropriate credit to the original author(s) and the source, provide a link to the Creative Commons license, and indicate if changes were made. 
this subject can be found in [2] for operators having a bounded domain and in [1] for non-surjective operators.

A different type of generalization consists in dealing with mixed-type operators, that is, equations of type

$$
\left(h(t, x) \Phi\left(x^{\prime}\right)\right)^{\prime}=g\left(t, x, x^{\prime}\right) \quad \text { or } \quad h(t, x)\left(\Phi\left(x^{\prime}\right)\right)^{\prime}=g\left(t, x, x^{\prime}\right)
$$

(see, e.g., [3-6, 9-11, 22]).

In recent papers (see $[20,21])$, some existence and non-existence results were proved for the boundary value problem

$$
\left\{\begin{array}{l}
\left(h(t, x(t)) \Phi\left(x^{\prime}(t)\right)\right)^{\prime}=g\left(t, x(t), x^{\prime}(t)\right) \quad \text { a.e. } t \in \mathbb{R}, \\
x(-\infty)=a, \quad x(+\infty)=b,
\end{array}\right.
$$

where $\Phi$ is a general increasing homeomorphism in $\mathbb{R}$. The generality of the differential operator $\Phi$ required a rather strong growth assumption on the right-hand side $g\left(t, x, x^{\prime}\right)$ with respect to $x^{\prime}$ (see conditions 3.3 and 3.4 in [20, Theorem 3.1]). For instance, when $g(t, x, y)=a(t) b(x) c(y)$ and $\Phi$ has a superlinear growth at infinity, then the condition $c\left(x^{\prime}\right) /\left|\Phi\left(x^{\prime}\right)\right| \rightarrow 0$ as $\left|x^{\prime}\right| \rightarrow \infty$ is needed (among others) in order to obtain the existence of solutions (see [20, Corollary 4.13]). So, in the special case of the $p$-Laplacian operator, the function $g\left(t, x, x^{\prime}\right)$ has to grow less than $p-1$ as $\left|x^{\prime}\right| \rightarrow+\infty$.

The aim of the present paper is to show that when dealing with the $p$-Laplacian operator, that is, when one has the following equation:

$$
\text { (P) }\left\{\begin{array}{l}
\left(h(t, x(t))\left|x^{\prime}(t)\right|^{p-2} x^{\prime}(t)\right)^{\prime}=g\left(t, x(t), x^{\prime}(t)\right) \quad \text { a.e. } t \in \mathbb{R}, \\
x(-\infty)=a, \quad x(+\infty)=b
\end{array}\right.
$$

the growth assumption on the right-hand side $g$ considered in [20] can be improved and the class of solvable problems can be widened. More in detail, when $g(t, x, y)=a(t) b(x) c(y)$, the assumption $c\left(x^{\prime}\right) /\left|\Phi\left(x^{\prime}\right)\right| \rightarrow 0$ as $\left|x^{\prime}\right| \rightarrow \infty$ can be replaced by $c(y)=O\left(|y|^{p}\right)$ (see condition (3.26)). To our knowledge, the existence result here presented is new also for the classical case $p=2$. For instance, as an application of our results, we have that the differential equation

$$
\left(|t|^{n} \beta(x) x^{\prime}(t)\right)^{\prime}=-t^{m} g(x)\left(x^{\prime}(t)\right)^{2},
$$

where $\beta(x), g(x)$ are generic positive continuous functions, admits solutions satisfying $x(-\infty)=a, x(+\infty)=b$ for every $a, b$ with $a<b$, provided that $m$ is odd and $m>2 n+1$ (see Example 3.7). We underline that the previous equation can not be treated by means of the results in [20], since in this case $p=2$ and the growth of $f$ with respect to $x^{\prime}$ is greater than $p-1$.

\section{Existence and non-existence theorem}

In the whole paper we will consider a positive continuous function $h: \mathbb{R} \times[a, b] \rightarrow \mathbb{R}$ and a Carathéodory function $g: \mathbb{R}^{3} \rightarrow \mathbb{R}$. 
We deal with the following nonlinear differential equation:

$$
\left(h(t, x(t))\left|x^{\prime}(t)\right|^{p-2} x^{\prime}(t)\right)^{\prime}=g\left(t, x(t), x^{\prime}(t)\right) \quad \text { a.e. } t
$$

and we use the following notations:

$$
\begin{aligned}
& m(t):=\min _{x \in[a, b]} h(t, x), \quad M(t):=\max _{x \in[a, b]} h(t, x), \\
& m^{*}(t):=\min _{(s, x) \in[-t, t] \times[a, b]} h(s, x), \quad M^{*}(t):=\max _{(s, x) \in[-t, t] \times[a, b]} h(s, x) .
\end{aligned}
$$

Of course, $M^{*}(t) \geq M(t) \geq m(t) \geq m^{*}(t)>0$ for every $t \in \mathbb{R}$, with inf $\operatorname{in}_{t \in \mathbb{R}} m(t)$ possibly zero.

Our main results are the following general existence and non-existence theorems.

Theorem 2.1 Suppose that

$$
g(t, a, 0) \leq 0 \leq g(t, b, 0) \quad \text { for a.e. } t \in \mathbb{R}
$$

and that there exist constants $C_{1}, C_{2}>0$, a continuous function $\mu: \mathbb{R}^{+} \rightarrow \mathbb{R}^{+}$, and a function $\lambda \in L^{q}\left(\left[-C_{1}, C_{1}\right]\right)$ with $1 \leq q \leq \infty$ such that

$$
\begin{aligned}
& |g(t, x, y)| \leq \lambda(t) \mu\left(h(t, x)|y|^{p-1}\right) \quad \text { for a.e. }|t| \leq C_{1}, \text { every } x \in[a, b],|y| \geq C_{2}, \\
& \int^{+\infty} \frac{\tau^{\frac{q-1}{q(p-1)}}}{\mu(\tau)} \mathrm{d} \tau=+\infty
\end{aligned}
$$

(with $\frac{q-1}{q(p-1)}=\frac{1}{p-1}$ if $\left.q=+\infty\right)$.

At last, suppose that there exists a constant $\gamma>1$ such that, for every $C>0$, there exists a function $\Lambda_{C} \in L_{\mathrm{loc}}^{1}([0,+\infty))$, null in $\left[0, C_{1}\right]$ and positive in $\left(C_{1},+\infty\right)$, such that:

$$
m(t)^{1-\gamma} \cdot\left(\int_{0}^{t} \frac{\Lambda_{C}(|s|)}{M(s)^{\gamma}} \mathrm{d} s\right)^{-1} \in L^{\frac{1}{(p-1)(\gamma-1)}}(\mathbb{R})
$$

and there exists a function $\eta_{C} \in L^{1}(\mathbb{R})$ such that putting

$$
N_{C}(t):=m(t)^{-\frac{1}{p-1}}\left\{\left(M^{*}\left(C_{1}\right) C^{p-1}\right)^{1-\gamma}+(\gamma-1) \int_{0}^{t} \frac{\Lambda_{C}(|s|)}{M(s)^{\gamma}} \mathrm{d} s\right\}^{-\frac{1}{\gamma-1} \frac{1}{p-1}}
$$

for every $x \in[a, b]$ and every $|y| \leq N_{C}(t)$, we have

$$
\begin{aligned}
& \left\{\begin{array}{l}
g(t, x, y) \leq-\Lambda_{C}(t)|y|^{\gamma(p-1)} \\
g(-t, x, y) \geq \Lambda_{C}(t)|y|^{\gamma(p-1)}
\end{array} \quad \text { for a.e. } t \geq C_{1},\right. \\
& |g(t, x, y)| \leq \eta_{C}(t) \quad \text { for a.e. } t \in \mathbb{R} .
\end{aligned}
$$


Then there exists a function $x \in C^{1}(\mathbb{R})$ such that $\left.t \mapsto h(t, x(t))\left|x^{\prime}(t)\right|^{p-1} x^{\prime}(t)\right)$ belongs to $W^{1,1}(\mathbb{R})$ and

$$
\begin{cases}\left(h(t, x(t))\left|x^{\prime}(t)\right|^{p-1} x^{\prime}(t)\right)^{\prime}=g\left(t, x(t), x^{\prime}(t)\right), & \text { for a.e. } t \in \mathbb{R}, \\ a \leq x(t) \leq b, & \text { for every } t \in \mathbb{R}, \\ x(-\infty)=a, & x(+\infty)=b .\end{cases}
$$

Proof With no restriction we may assume $C_{2}>\frac{1}{2 C_{1}}(b-a)$. By (2.5) there exists a constant $C$ such that

$$
C>\left(\frac{M^{*}\left(C_{1}\right)}{m^{*}\left(C_{1}\right)}\right)^{\frac{1}{p-1}} C_{2} \geq C_{2}
$$

and

$$
\int_{M^{*}\left(C_{1}\right) C_{2}^{p-1}}^{m^{*}\left(C_{1}\right) C^{p-1}} \frac{\tau^{\frac{q-1}{q(p-1)}}}{\mu(\tau)} \mathrm{d} \tau>\|\lambda\|_{q}\left[M^{*}\left(C_{1}\right)^{\frac{1}{p-1}}(b-a)\right]^{1-\frac{1}{q}} .
$$

Fix $n \in \mathbb{N}, n>C_{1}$, and put $I_{n}:=[-n, n]$. Consider the truncation operator $U: W^{1,1}\left(I_{n}\right) \rightarrow$ $W^{1,1}\left(I_{n}\right)$ defined by

$$
U(x):=U_{x}, \quad \text { where } U_{x}(t):=\max \{a, \min \{b, x(t)\}\}
$$

and for every $x \in W_{\text {loc }}^{1,1}(\mathbb{R})$, put

$$
V_{x}(t):=\max \left\{-N_{C}(t), \min \left\{U_{x}^{\prime}(t), N_{C}(t)\right\}\right\} .
$$

Moreover, for every $x \in \mathbb{R}$, put $w(x):=\max \{x-b, 0\}+\min \{x-a, 0\}$.

Let us consider the following auxiliary boundary value problem on the compact interval $I_{n}$ :

$$
\left(P_{n}^{*}\right) \quad\left\{\begin{array}{l}
\left(h\left(t, U_{x}(t)\right)\left|x^{\prime}(t)\right|^{p-2} x^{\prime}(t)\right)^{\prime}=g\left(t, U_{x}(t), V_{x}(t)\right)+\frac{w(x(t))}{|w(x(t))|+1}, \quad \text { a.e. in } I_{n}, \\
x(-n)=a, \quad x(n)=b .
\end{array}\right.
$$

By the same argument developed in the proof of [20, Theorem 3.1], it is possible to prove that problem (2.13) admits a solution $u_{n}$ for every $n>C_{1}$ such that $a \leq u_{n}(t) \leq b$ for all $t \in I_{n}$, so that $U_{u_{n}}(t)=u_{n}(t)$ for every $t \in I_{n}$. Moreover, $u_{n}$ is increasing in $\left[-n,-C_{1}\right]$ and in $\left[C_{1}, n\right]$ and if $u_{n}^{\prime}\left(t_{0}\right)=0$ for some $C_{1}<\left|t_{0}\right|<n$, then $u_{n}^{\prime}(t)=0$ whenever $|t|>\left|t_{0}\right|$ (see Steps $1-2$ in the proof of [20, Theorem 3.1]).

Now our goal is to prove that $\left|u_{n}^{\prime}(t)\right| \leq N_{C}(t)$ for every $t \in I_{n}$, so that also $V_{u_{n}}(t)=u_{n}^{\prime}(t)$ in $I_{n}$ and consequently $u_{n}$ is a solution of equation (2.1) too.

To this aim, put

$$
f(t):=h\left(t, u_{n}(t)\right) u_{n}^{\prime}(t)\left|u_{n}^{\prime}(t)\right|^{p-2} \quad \text { in } I_{n},
$$


we claim that

$$
|f(t)| \leq m^{*}\left(C_{1}\right) C^{p-1} \quad \text { for every } t \in\left[-C_{1}, C_{1}\right]
$$

implying that $\left|u_{n}^{\prime}(t)\right| \leq C$ for every $t \in\left[-C_{1}, C_{1}\right]$.

Indeed, notice that by the Lagrange theorem there exists a point $\tau_{0} \in I_{n}$ such that

$$
\left|u_{n}^{\prime}\left(\tau_{0}\right)\right|=\frac{1}{2 C_{1}}\left|u_{n}\left(C_{1}\right)-u_{n}\left(-C_{1}\right)\right| \leq \frac{b-a}{2 C_{1}}<C_{2}<C,
$$

so, by (2.11), we have

$$
\left|f\left(\tau_{0}\right)\right| \leq M^{*}\left(C_{1}\right) C_{2}^{p-1}<m^{*}\left(C_{1}\right) C^{p-1} .
$$

Assume, by contradiction, the existence of an interval $\left(\tau_{1}, \tau_{2}\right) \subset\left(-C_{1}, C_{1}\right)$ such that $|f(t)|<$ $m^{*}\left(C_{1}\right) C^{p-1}$ in $\left(\tau_{1}, \tau_{2}\right)$ and $\left|f\left(\tau_{1}\right)\right|=M^{*}\left(C_{1}\right) C_{2}^{p-1},\left|f\left(\tau_{2}\right)\right|=m^{*}\left(C_{1}\right) C^{p-1}$ or vice versa.

Then we have $C_{2} \leq u_{n}^{\prime}(t) \leq C$ in $\left(\tau_{1}, \tau_{2}\right)$ and since $N_{C}(t)=C\left(\frac{M^{*}\left(C_{1}\right)}{m(t)}\right)^{\frac{1}{p-1}} \geq C$ for every $t \in\left(-C_{1}, C_{1}\right)$, we have $\left|u_{n}^{\prime}(t)\right|<N_{C}(t)$ for every $t \in\left(\tau_{1}, \tau_{2}\right)$. Then, by Step 1, the definition of $\left(P_{n}^{*}\right)$, and assumption (2.4), for a.e. $t \in\left(\tau_{1}, \tau_{2}\right)$, we have

$$
\left|f^{\prime}(t)\right|=\left|g\left(t, U_{u_{n}}(t), V_{u_{n}}(t)\right)\right| \leq \lambda(t) \mu(|f(t)|) .
$$

Therefore, by the Hölder inequality we get

$$
\begin{aligned}
\int_{M^{*}\left(C_{1}\right) C_{2}^{p-1}}^{m^{*}\left(C_{1}\right) C^{p-1}} \frac{\tau^{\frac{q-1}{q(p-1)}}}{\mu(\tau)} \mathrm{d} \tau & \leq \int_{\tau_{1}}^{\tau_{2}} \frac{|f(t)| \frac{q-1}{q(p-1)}}{\mu(|f(t)|)}\left|f^{\prime}(t)\right| \mathrm{d} t \\
& \leq \int_{\tau_{1}}^{\tau_{2}} \lambda(t) h\left(t, u_{n}(t)\right)^{\frac{q-1}{q(p-1)}}\left|u_{n}(t)\right|^{1-\frac{1}{q}} \mathrm{~d} t \\
& \leq\|\lambda\|_{q} M^{*}\left(C_{1}\right)^{\frac{q-1}{q(p-1)}}\left(\int_{\tau_{1}}^{\tau_{2}}\left|u_{n}^{\prime}(t)\right| \mathrm{d} t\right)^{1-\frac{1}{q}} \\
& \leq\|\lambda\|_{q}\left(M^{*}\left(C_{1}\right)^{\frac{1}{p-1}} \int_{\tau_{1}}^{\tau_{2}}\left|u_{n}^{\prime}(t)\right| \mathrm{d} t\right)^{1-\frac{1}{q}} \\
& \leq\|\lambda\|_{q}\left[M^{*}\left(C_{1}\right)^{\frac{1}{p-1}}(b-a)\right]^{1-\frac{1}{q}}
\end{aligned}
$$

in contradiction with (2.12). Thus, claim (2.14) is proved, and consequently we have $\left|u_{n}^{\prime}(t)\right|<C \leq N_{C}(t)$ for every $t \in\left[-C_{1}, C_{1}\right]$.

We now prove that $u_{n}^{\prime}(t) \leq N_{C}(t)$ for every $t \in I_{n} \backslash\left[-C_{1}, C_{1}\right]$.

To this aim, let $\hat{t}:=\sup \left\{t>C_{1}: u_{n}^{\prime}(\tau)<N_{C}(\tau)\right.$ in $\left.\left[C_{1}, t\right]\right\}$. Assume, by contradiction, $\hat{t}<n$. By the definition of $V_{u_{n}}$, we have

$$
\left(h\left(t, u_{n}(t)\right) u_{n}^{\prime}(t)^{p-1}\right)^{\prime}=g\left(t, U_{u_{n}}(t), V_{u_{n}}(t)\right)=g\left(t, u_{n}(t), u_{n}^{\prime}(t)\right) \quad \text { a.e. in }\left[C_{1}, \hat{t}\right] .
$$

Recalling that $u_{n}^{\prime}(t) \geq 0$ in $\left[C_{1}, n\right)$, by $(2.8)$ we have

$$
\left(h\left(t, u_{n}(t)\right) u_{n}^{\prime}(t)^{p-1}\right)^{\prime} \leq-\Lambda_{C}(t) u_{n}(t)^{\gamma(p-1)} \leq-\frac{\Lambda_{C}(t)}{M(t)^{\gamma}}\left[h\left(t, u_{n}(t)\right) u_{n}^{\prime}(t)^{p-1}\right]^{\gamma}
$$


for a.e. $t \in\left[C_{1}, \hat{t}\right]$. Then

$$
\begin{aligned}
& \frac{1}{1-\gamma}\left\{\left[h\left(t, u_{n}(t)\right) u_{n}^{\prime}(t)^{p-1}\right]^{1-\gamma}-\left[h\left(C_{1}, u_{n}\left(C_{1}\right)\right) u_{n}^{\prime}\left(C_{1}\right)^{p-1}\right]^{1-\gamma}\right\} \\
& =\int_{C_{1}}^{t} \frac{\left(h\left(u_{n}(s)\right) u_{n}^{\prime}(s)^{p-1}\right)^{\prime}}{\left(h\left(u_{n}(s)\right) u_{n}^{\prime}(s)^{p-1}\right)^{\gamma}} \mathrm{d} s \leq-\int_{C_{1}}^{t} \frac{\Lambda_{C}(s)}{M(s)^{\gamma}} \mathrm{d} s=-\int_{0}^{t} \frac{\Lambda_{C}(s)}{M(s)^{\gamma}} \mathrm{d} s
\end{aligned}
$$

for every $t \in\left[C_{1}, \bar{t}\right]$. Therefore,

$$
\begin{aligned}
\left(h\left(t, u_{n}(t)\right) u_{n}^{\prime}(t)^{p-1}\right)^{1-\gamma} & \geq\left(h\left(C_{1}, u_{n}\left(C_{1}\right)\right) u_{n}^{\prime}\left(C_{1}\right)^{p-1}\right)^{1-\gamma}+(\gamma-1) \int_{0}^{t} \frac{\Lambda_{C}(s)}{M(s)^{\gamma}} \mathrm{d} s \\
& >\left(M^{*}\left(C_{1}\right) C^{p-1}\right)^{1-\gamma}+(\gamma-1) \int_{0}^{t} \frac{\Lambda_{C}(s)}{M(s)^{\gamma}} \mathrm{d} s
\end{aligned}
$$

implying that

$$
u_{n}^{\prime}(t)<\left(\frac{1}{m(t)}\left\{\left(M^{*}\left(C_{1}\right) C^{p-1}\right)^{1-\gamma}+(\gamma-1) \int_{0}^{t} \frac{\Lambda_{C}(s)}{M(s)^{\gamma}} \mathrm{d} s\right\}^{\frac{1}{1-\gamma}}\right)^{\frac{1}{p-1}}=N_{C}(t)
$$

for every $t \in\left[C_{1}, \hat{t}\right]$, a contradiction when $\hat{t}<n$. So, $\hat{t}=n$ and the claim is proved. The same argument works in the interval $\left[-n,-C_{1}\right]$ too.

Therefore, we have $\left|u_{n}^{\prime}(t)\right| \leq N_{C}(t)$ for every $t \in[-n, n]$ implying that

$$
h\left(t, u_{n}(t)\right)\left|u_{n}^{\prime}(t)\right|^{p-2} u_{n}^{\prime}(t)=g\left(t, u_{n}(t), u_{n}^{\prime}(t)\right) \quad \text { a.e. in } I_{n} .
$$

Now, following the same argument as in [20, Theorem 3.1], one can show that the sequence $\left(\tilde{u}_{n}\right)_{n}$ of the functions $u_{n}$ continued in a constant way in the whole $\mathbb{R}$ converges to a solution $x$ of problem (2.10), satisfying all the properties stated in the assertion.

The main tool in the previous existence theorem is the summability of function $N_{C}(t)$ (condition (2.6)) combined with assumption (2.8). Such conditions are not improvable in the sense that if (2.8) is satisfied with the reversed inequality and $N_{C}$ is not summable, then problem $(P)$ does not admit solutions, as stated in the following result.

Theorem 2.2 Suppose that there exist three constants $C_{1} \geq 0, \rho>0, \gamma>1$ and a positive function $\Lambda \in L_{\text {loc }}^{1}\left(\left[C_{1},+\infty\right)\right)$ such that one of the following pairs of conditions holds:

$$
g(t, x, y) \geq-\Lambda(t) y^{\gamma(p-1)} \quad \text { for a.e. } t \geq C_{1} \text {, every } x \in[a, b], y \in(0, \rho)
$$

or

$$
g(t, x, y) \leq \Lambda(-t) y^{\gamma(p-1)} \quad \text { for a.e. } t \leq-C_{1} \text {, every } x \in[a, b], y \in(0, \rho)
$$

and for every constant $C$, the function

$$
N_{C}(t):=\left(\frac{1}{M(t)}\left\{C+(\gamma-1) \int_{C_{1}}^{t} \frac{\Lambda(s)}{m(s)^{\gamma}} \mathrm{d} s\right\}^{\frac{1}{1-\gamma}}\right)^{\frac{1}{p-1}}
$$

does not belong to $L^{1}([1,+\infty))$. 
Moreover, assume that

$$
\operatorname{tg}(t, x, y) \leq 0 \quad \text { for a.e. }|t| \geq C_{1} \text {, every }(x, y) \in[a, b] \times \mathbb{R},
$$

and there exist two constants $k, C_{2}>0$ such that

$$
\begin{array}{ll}
h\left(t, x_{1}\right) \leq C_{2} h\left(t+\delta, x_{2}\right) & \text { for every } t>C_{1}, x_{1}, x_{2} \in[a, b] \text { and } \delta<k, \\
h\left(t+\delta, x_{1}\right) \leq C_{2} h\left(t, x_{2}\right) & \text { for every } t<-C_{1}, x_{1}, x_{2} \in[a, b] \text { and } \delta<k .
\end{array}
$$

Then each possible solution $x$ of problem $(P)$ is constant in $\left[C_{1},+\infty\right)$ (when (2.15) holds) or constant in $\left(-\infty,-C_{1}\right]$ (when (2.16) holds).

Therefore, if both (2.15) and (2.16) hold and $C_{1}=0$, then problem $(P)$ does not admit solutions, that is, there exists no function $x \in C^{1}(\mathbb{R})$ such that $t \mapsto h(t, x(t))\left|x^{\prime}(t)\right|^{p-2} x^{\prime}(t)$ is almost everywhere differentiable, satisfying the conditions of problem $(P)$.

Remark 2.1 Of course, (2.19), (2.20) are satisfied (for $C_{1}=0$ ) when $h(t, x)=h_{1}(t) h_{2}(x)$, provided that $h_{1}(t)$ is decreasing in $(-\infty, 0)$ and increasing in $(0,+\infty)$, or $h_{1}$ is uniformly continuous in $\mathbb{R}$ and $\inf _{t \in \mathbb{R}} h_{1}(t)>0$, or $h_{1}(t) \sim|t|^{-k}$ as $|t| \rightarrow+\infty$ for some $k>0$, since $h_{2}(x)>0$ on $[a, b]$.

\section{Asymptotic criteria}

We now provide some applications for operators and right-hand side having the product structure

$$
h(t, x)=h_{1}(t) h_{2}(x) \quad \text { and } \quad g(t, x, y)=g_{1}(t, x) g_{2}(x, y) .
$$

We emphasize the link between the local behavior of $g_{2}(x, \cdot)$ at $y=0$ and of $g_{1}(\cdot, x), h_{1}(\cdot)$ at infinity, which is crucial for the existence or non-existence of solutions.

In what follows we assume that $h_{1}, h_{2}$ are continuous positive functions, $g_{1}$ is a Carathéodory function, and $g_{2}$ is a continuous function satisfying

$$
g_{2}(x, y)>0 \quad \text { for every } y \neq 0 \text { and } x \in[a, b] ; \quad g_{2}(a, 0)=g_{2}(b, 0)=0 \text {. }
$$

Put $\tilde{m}:=\min _{x \in[a, b]} h_{2}(x)$ and $\tilde{M}:=\max _{x \in[a, b]} h_{2}(x)$, we have

$$
m(t)=\tilde{m} h_{1}(t) \quad \text { and } \quad M(t)=\tilde{M} h_{1}(t) \quad \text { for every } t \in \mathbb{R},
$$

where recall that $m(t):=\min _{x \in[a, b]} h(t, x)$ and $M(t):=\max _{x \in[a, b]} h(t, x)$.

Moreover, from now on we put

$$
m_{\infty}:=\inf _{t \in \mathbb{R}} h_{1}(t) \geq 0 .
$$

The following existence theorems are application of Theorem 2.1.

Proposition 3.1 Suppose that, for some $C_{1}>0$, we have

$$
t \cdot g_{1}(t, x)<0 \quad \text { for a.e. } t \text { such that }|t| \geq C_{1} \text {, every } x \in[a, b],
$$


and there exists a function $\lambda \in L_{\mathrm{loc}}^{q}(\mathbb{R}), 1 \leq q \leq+\infty$, such that

$$
\left|g_{1}(t, x)\right| \leq \lambda(t) \quad \text { for a.e. } t \in \mathbb{R} \text {, every } x \in[a, b]
$$

Moreover, assume that there exist real constants $\sigma, \delta$, and $\gamma>1$ satisfying one of the following pairs of conditions:

$$
\begin{array}{ll}
\delta+1>\sigma \gamma, & (p-1)(\gamma-1)<\delta+1-\sigma, \\
\delta+1<\sigma \gamma, & \sigma>p-1,
\end{array}
$$

such that, for every $x \in[a, b]$, we have

$$
\begin{aligned}
& h_{1}|t|^{\sigma} \leq h_{1}(t) \leq h_{2}|t|^{\sigma}, \quad \text { a.e. }|t|>C_{1}, \\
& h_{1}|t|^{\delta} \leq\left|g_{1}(t, x)\right| \leq h_{2}|t|^{\delta}, \quad \text { a.e. }|t|>C_{1}, \\
& g_{2}(x, y) \leq k_{2}|y|^{\gamma(p-1)} \quad \text { whenever }|y|<\rho, \\
& g_{2}(x, y) \leq k_{2}|y|^{p-\frac{1}{q}} \quad \text { whenever }|y|>C_{2}, \\
& g_{2}(x, y) \geq k_{1}|y|^{\gamma(p-1)} \quad \text { for every } y \in \mathbb{R} .
\end{aligned}
$$

for certain positive constants $h_{1}, h_{2}, k_{1}, k_{2}, \rho, C_{2}$.

Then problem $(P)$ admits solutions.

Proof It is not restrictive to assume $C_{2}>\max \left\{C_{1}, \frac{b-a}{2 C_{1}}\right\}$. Put $\mu(r):=k_{2}\left(\frac{r}{m^{*}\left(C_{1}\right)}\right)^{\frac{q p-1}{q(p-1)}}$ for $r>0$ (see (2.2)), from (3.3) and (3.9) the validity of conditions (2.4) and (2.5) follows.

Put $\Lambda(t):=0$ for $0 \leq t \leq C_{1}$, and

$$
\Lambda(t):=k_{1} \min \left\{\min _{x \in[a, b]} g_{1}(-t, x), \min _{x \in[a, b]}-g_{1}(t, x)\right\} \quad \text { for } t \geq C_{1}
$$

By condition (3.3) we have $\Lambda \in L_{\text {loc }}^{1}([0,+\infty))$, and by (3.2) we have that $\Lambda$ is positive. Observe that by (3.10) it follows that

$$
g(t, x, y)=g_{1}(t, x) g_{2}(x, y) \leq k_{1} g_{1}(t, x)|y|^{\gamma(p-1)} \leq-\Lambda(t)|y|^{\gamma(p-1)}
$$

and

$$
g(-t, x, y)=g_{1}(-t, x) g_{2}(x, y) \geq k_{1} g_{1}(-t, x)|y|^{\gamma(p-1)} \geq \Lambda(t)|y|^{\gamma(p-1)}
$$

for a.e. $t \geq C_{1}$, every $x \in[a, b]$, and every $y \in \mathbb{R}$. Then condition (2.8) of Theorem 2.1 holds, with $\Lambda_{C}(\cdot):=\Lambda(\cdot)$ for every $C>0$.

Now, from (3.7) it follows that $h_{1} k_{1} t^{\delta} \leq \Lambda(t)$ for a.e. $t \geq C_{1}$ and by (3.6) we deduce that, for some positive constant $c_{1}$, we have

$$
\int_{0}^{t} \frac{\Lambda(|s|)}{h_{1}(s)^{\gamma}} \mathrm{d} s=\int_{C_{1}}^{t} \frac{\Lambda(|s|)}{h_{1}(s)^{\gamma}} \mathrm{d} s \geq c_{1}|t|^{\delta-\gamma \sigma+1} \quad \text { for every } t \text { large enough. }
$$


Hence, by condition (3.6) we obtain

$$
m(t)^{1-\gamma}\left(\int_{0}^{t} \frac{\Lambda(|s|)}{h_{1}(s)^{\gamma}} \mathrm{d} s\right)^{-1} \leq c_{2}|t|^{-(\delta+1-\sigma)}
$$

for some positive constant $c_{2}$, implying by the second inequality in (3.4) the validity of assumption (2.6). Moreover, the function $N_{C}(t)$ defined in (2.7) satisfies

$$
N_{C}(t)^{p-1} \leq c_{3}|t|^{\frac{\delta+1-\sigma \gamma}{1-\gamma}-\sigma}=c_{3}|t|^{\frac{\delta+1-\sigma}{1-\gamma}} \quad \text { for } t \text { large enough }
$$

for some constant $c_{3}$. So, by the first inequality in (3.4) we have $\lim _{|t| \rightarrow+\infty} N_{C}(t)=0$, and then a constant $L_{C}^{*}>C_{1}$ exists such that $N_{C}(t) \leq \rho$ for every $|t| \geq L_{C}^{*}$. Let us define $\hat{C}:=$ $\max _{|t| \leq L_{C}^{*}} N_{C}(t)$ and

$$
\eta_{C}(t):= \begin{cases}\max _{x \in[a, b]}\left|g_{1}(t, x)\right| \cdot \max _{(x, y) \in[a, b] \times[-\hat{C}, \hat{C}]} g_{2}(x, y) & \text { if }|t| \leq L_{C}^{*} \\ h_{2} k_{2}|t|^{\delta} N_{C}(t)^{\gamma(p-1)} & \text { if }|t|>L_{C}^{*}\end{cases}
$$

By (3.7) and (3.8), for a.e. $t \in \mathbb{R}$, for every $x \in[a, b]$ and every $y \in \mathbb{R}$ such that $|y| \leq N_{C}(t)$, we have

$$
|g(t, x, y)|=\left|g_{1}(t, x)\right| g_{2}(x, y) \leq \eta_{C}(t)
$$

so it remains to prove that $\eta_{C} \in L^{1}(\mathbb{R})$.

By (3.3) and the continuity of the function $g_{2}(\cdot, \cdot)$, we have $\eta_{C} \in L^{1}\left(\left[-L_{C}^{*}, L_{C}^{*}\right]\right)$. Moreover, when $|t|>L_{C}^{*}$, by (3.12) we have

$$
\eta_{C}(t) \leq \text { Const. }|t|^{\delta+\gamma \frac{\delta+1-\sigma}{1-\gamma}}=\text { Const. }|t|^{\frac{\delta+\gamma-\sigma \gamma}{1-\gamma}}
$$

implying that $\eta_{C}(t) \in L^{1}\left(\mathbb{R} \backslash\left[-L_{C}^{*}, L_{C}^{*}\right]\right)$ by the first condition in (3.4).

Therefore, we can apply Theorem 2.1 and obtain the assertion of the present result.

The case of (3.5) is similar.

If $\Lambda$ is the function defined above, by the first inequality in (3.5) we get that the integral function $t \mapsto \int_{0}^{t} \frac{\Lambda(|s|)}{M(s)^{\gamma}} \mathrm{d} s$ is bounded. So, the second condition in (3.5) implies the validity of assumption (2.6). Moreover, if $\eta_{C}$ is defined as above, then

$$
\eta_{C}(t) \sim \text { Const. }|t|^{\delta} \frac{1}{h_{1}(t)^{\gamma}} \leq \text { Const. } t^{\delta-\sigma \gamma} \quad \text { as } t \rightarrow+\infty
$$

so $\eta_{C}$ is summable by condition (3.5) and the proof proceeds as in the first part.

Remark 3.1 We underline that conditions (3.9) and (3.10) are compatible with each other for large $|y|$ only if $q>1$ and $\gamma \leq \frac{p-\frac{1}{q}}{p-1}$. However, if $m_{\infty}>0$ (see (3.1)), condition (3.10) can be improved requiring that it holds only for $|y|$ small enough, as the following result states.

Proposition 3.2 Let all the assumption of Proposition 3.1 be satisfied, with the exception of (3.10), replaced by

$$
g_{2}(x, y) \geq k_{1}|y|^{\gamma(p-1)} \quad \text { for every } x \in[a, b],|y|<\rho .
$$

Moreover, assume that $m_{\infty}>0$. Then problem $(P)$ admits solutions. 
Proof For every fixed $C>0$, let

$$
\Gamma_{C}:=\max \left\{\rho, C\left(\frac{M^{*}\left(C_{1}\right)}{m_{\infty}}\right)^{\frac{1}{p-1}}\right\}, \quad \hat{m}_{C}:=\min _{(x, y) \in[a, b] \times\left[\rho, \Gamma_{C}\right]} g_{2}(x, y),
$$

and finally

$$
h_{C}:=\min \left\{k_{1}, \frac{\hat{m}_{C}}{\Gamma_{C}^{\gamma(p-1)}}\right\} .
$$

Let us define the function $\Lambda(t)$ as in the proof of Proposition (3.1), with $k_{1}$ replaced by $h_{C}$.

Notice that $N_{C}(t) \leq \Gamma_{C}$ for every $t \geq C_{1}$; moreover $g_{2}(x, y) \geq h_{C}|y|^{\gamma(p-1)}$ whenever $|y| \leq$ $\Gamma_{C}$. So, condition (2.8) holds whenever $|y|<\Gamma_{C}$ and the proof proceeds as that of Proposition 3.1, replacing everywhere $k_{1}$ with $h_{C}$.

We state now two non-existence results, obtained applying Theorem 2.2.

Proposition 3.3 Suppose that

$$
t \cdot g_{1}(t, x) \leq 0 \quad \text { for a.e. } t \in \mathbb{R} \text { and every } x \in[a, b]
$$

and let there exist real constants $\delta, \gamma>1, \Lambda>0$ and a positive function $\ell(t) \in L^{1}([0, \Lambda])$ such that

$$
\begin{aligned}
& \left|g_{1}(t, x)\right| \leq \lambda_{1}|t|^{\delta} \quad \text { for every } x \in[a, b], \text { a.e. }|t|>\Lambda, \\
& \left|g_{1}(t, x)\right| \leq \ell(|t|) \quad \text { for a.e. }|t| \leq \Lambda, x \in[a, b], \\
& g_{2}(x, y) \leq \lambda_{2} y^{\gamma(p-1)} \quad \text { for every } x \in[a, b], 0<y<\rho
\end{aligned}
$$

for some positive constants $\lambda_{1}, \lambda_{2}, \rho$. Moreover, assume that (3.6) holds for some constants $h_{1}, h_{2}, \sigma$ such that one of the following pairs of conditions is satisfied:

$$
\begin{aligned}
& \delta+1>\sigma \gamma, \quad(p-1)(\gamma-1) \geq \delta+1-\sigma, \\
& \delta+1 \leq \sigma \gamma, \quad \sigma \leq p-1 .
\end{aligned}
$$

At last, suppose that there exist two constants $\epsilon, C_{2}>0$ such that

$$
\begin{aligned}
& h_{1}(t) \leq C_{2} h_{1}(t+r) \quad \text { for every } t>0 \text { and } r<\epsilon \\
& h_{1}(t+r) \leq C_{2} h_{1}(t) \quad \text { for every } t<0 \text { and } r<\epsilon .
\end{aligned}
$$

Then problem $(P)$ does not admit solutions.

Proof Put

$$
\Lambda(t):= \begin{cases}\lambda_{2} \ell(t) & \text { for } t \in[0, \Lambda] \\ \lambda_{1} \lambda_{2} t^{\delta} & \text { for } t>\Lambda\end{cases}
$$


we have that $\Lambda$ is a positive function belonging to $L_{\text {loc }}^{1}([0,+\infty))$ and one can easily verify that conditions (3.15), (3.16), and (3.17) ensure the validity of (2.15) and (2.16) with $C_{1}=0$. Moreover, by (3.6) and the very definition of $\Lambda$, one has

$$
\begin{aligned}
\rho(t) & :=\int_{0}^{t} \frac{\Lambda(\tau)}{m(\tau)^{\gamma}} \mathrm{d} \tau \geq \int_{\Lambda}^{t} \frac{\Lambda(\tau)}{m(\tau)^{\gamma}} \mathrm{d} \tau=\frac{\lambda_{1} \lambda_{2}}{\tilde{m}^{\gamma}} \int_{\Lambda}^{t} \frac{\tau^{\delta}}{h_{1}(\tau)^{\gamma}} \mathrm{d} \tau \\
& \geq \frac{\lambda_{1} \lambda_{2}}{\tilde{m}^{\gamma} h_{1}^{\gamma}} \int_{\Lambda}^{t} \tau^{\delta-\gamma \delta} \mathrm{d} \tau=\text { Const. } t^{\delta-\sigma \gamma+1} \quad \text { (provided } t \text { is sufficiently large). }
\end{aligned}
$$

As a consequence, for every constant $C$, we have

$$
\begin{aligned}
N_{C}(t) & =\left(\frac{1}{M(t)[C+(\gamma-1) \rho(t)]^{\frac{1}{\gamma-1}}}\right)^{\frac{1}{p-1}} \\
& \geq \text { Const. }\left(\frac{1}{t^{\sigma+\frac{\delta-\sigma \gamma+1}{\gamma-1}}}\right)^{\frac{1}{p-1}}=\text { Const. } t^{-\frac{\delta+1-\sigma}{(p-1)(\gamma-1)}},
\end{aligned}
$$

provided $t \in(0,+\infty)$ is sufficiently large. At last, the second assumption in (3.18) implies that $N_{C}(t)$ is not summable in $[1,+\infty)$ and the assertion follows as an application of Theorem 2.2 in the case $C_{1}=0$.

The case of (3.19) is similar.

By using the same notations, notice that under the first condition in (3.19) the integral function $\rho(t)=\int_{0}^{t} \frac{\Lambda(\tau)}{m(\tau) \gamma} \mathrm{d} \tau$ is bounded, hence $N_{C}(t)^{p-1} \geq$ Const. $t^{-\sigma}$, implying that $N_{C}(t) \geq$ Const. $t^{-\sigma /(p-1)}$. Therefore $N_{C}$ is not summable at infinity owing to the second condition in assumption (3.19), and the assertion follows from Theorem 2.2, applied for $C_{1}=0$.

As an immediate application of the previous theorems, the following criteria hold.

Corollary 3.4 Let $g(t, x, y)=g_{1}(t) g_{2}(x) g_{3}(y)$, where $g_{1} \in L_{\text {loc }}^{q}(\mathbb{R})$ for some $1 \leq q \leq+\infty$, $g_{3}$ is continuous in $\mathbb{R}$, and $g_{2}$ is continuous and positive in $[a, b]$.

Assume that $g_{3}(y)>0$ for $y \neq 0 ; t \cdot g_{1}(t) \leq 0$ for every $t$ and suppose that there exist constants $c_{1}, \ldots, c_{3}>0$ such that

$$
\begin{aligned}
& h_{1}(t) \sim c_{1}|t|^{\sigma} \text { as }|t| \rightarrow+\infty \quad \text { for some } \sigma \in \mathbb{R}, \\
& \left|g_{1}(t)\right| \sim c_{2}|t|^{\delta} \text { as }|t| \rightarrow+\infty \quad \text { for some } \delta \in \mathbb{R}, \\
& g_{3}(y) \sim c_{3}|y|^{\beta} \text { as } y \rightarrow 0 \quad \text { for some } \beta>0,
\end{aligned}
$$

with

$$
\delta+1>\frac{\sigma \beta}{p-1} \text { and } \beta>p-1 .
$$

Then, if conditions (3.20), (3.21) hold and $p \leq \beta+\sigma-\delta$, (P) has no solution.

Vice versa, if $p>\beta+\sigma-\delta$ and

$$
\limsup _{|y| \rightarrow+\infty} g_{3}(y) / y^{p-\frac{1}{q}} \in[0,+\infty)
$$




$$
g_{3}(y) \geq k_{1}|y|^{\beta} \quad \forall y \in \mathbb{R}
$$

then $(P)$ admits solutions.

Proof The assertion is an immediate consequence of Propositions 3.1 and 3.3 taking $\gamma=$ $\beta /(p-1)$.

Taking into account what we have observed in Remark 3.1, the following result holds in the particular case $m_{\infty}>0$.

Corollary 3.5 Let all the assumption of Corollary 3.4 hold, with the exception of (3.27). Then, if $m_{\infty}>0$, problem $(P)$ admits solutions.

When assumption (3.25) is not satisfied, we can use the following result, the consequence of Propositions 3.1 and 3.3.

Corollary 3.6 Let all the assumptions of Corollary 3.4 be satisfied, with the exception of (3.25), that is, assume that

$$
\delta+1<\frac{\sigma \beta}{p-1} .
$$

Then, if conditions (3.20), (3.21) hold and $\sigma+1 \leq p<\beta+1,(P)$ has no solution.

Vice versa, if $p<\sigma+1, p<\beta+1$, and we further assume (3.26) and (3.27), then (P) admits solutions.

At last, a result analogous to Corollary 3.6 holds when condition (3.27) is removed, provided that $m_{\infty}>0$, as in Corollary 3.5.

Example 3.7 Let us consider the following differential equation:

$$
\left(\left(1+|t|^{n}\right) \beta(x)\left|x^{\prime}(t)\right|^{p-2} x^{\prime}(t)\right)^{\prime}=-t^{m} g(x)\left(x^{\prime}(t)\right)^{2},
$$

where $\beta, g$ are generic positive continuous functions. By virtue of Corollary 3.4 taking $q=\infty, \sigma=n, \delta=m, s=2$, with $m>2 n-1$, we deduce that the differential equations admit solutions satisfying $x(-\infty)=a, x(+\infty)=b$, for any pair of ordered data $a, b$.

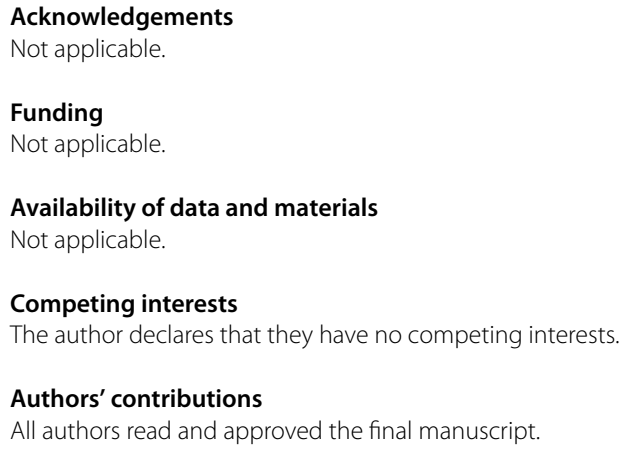




\section{Publisher's Note}

Springer Nature remains neutral with regard to jurisdictional claims in published maps and institutional affiliations.

Received: 7 August 2019 Accepted: 4 November 2019 Published online: 15 November 2019

\section{References}

1. Bereanu, C., Mawhin, J.: Boundary-value problems with non-surjective $\Phi$-Laplacian and one-side bounded nonlinearity. Adv. Differ. Equ. 11, 35-60 (2006)

2. Bereanu, C., Mawhin, J.: Existence and multiplicity results for some nonlinear problems with singular $\Phi$-Laplacian. J. Differ. Equ. 243, 536-557 (2007)

3. Biagi, S.: On the existence of weak solutions for singular strongly nonlinear boundary value problems on the half-line. Ann. Mat. Pura Appl. (2019). https://doi.org/10.1007/s10231-019-00893-2

4. Biagi, S., Calamai, A., Papalini, F.: Existence results for boundary value problems associated with singular strongly nonlinear equations. Submitted

5. Biagi, S., Calamai, A., Papalini, F.: Heteroclinic solutions for a class of boundary value problems associated with singular equations. Nonlinear Anal. To appear

6. Biagi, S., Isernia, T: On the solvability of singular boundary value problems on the real line in the critical growth case. Discrete Contin. Dyn. Syst. To appear

7. Cabada, A., Pouso, R.L.: Existence results for the problem $\left(\phi\left(u^{\prime}\right)\right)^{\prime}=g\left(t, u, u^{\prime}\right)$ with periodic and Neumann boundary conditions. Nonlinear Anal. 30, 1733-1742 (1997)

8. Cabada, A., Pouso, R.L.: Existence results for the problem $\left(\phi\left(u^{\prime}\right)\right)^{\prime}=g\left(t, u, u^{\prime}\right)$ with nonlinear boundary conditions. Nonlinear Anal. 35, 221-231 (1999)

9. Calamai, A., Marcelli, C., Papalini, F.: Boundary value problems for singular second order equations. Fixed Point Theory Appl. 2018, 20 (2018)

10. Cupini, G., Marcelli, C., Papalini, F.: Heteroclinic solutions of boundary value problems on the real line involving general nonlinear differential operators. Differ. Integral Equ. 24(7-8), 619-644 (2011)

11. Cupini, G., Marcelli, C., Papalini, F.: On the solvability of a boundary value problem on the real line. Bound. Value Probl. 2011, 26 (2011)

12. Dang, H., Oppenheimer, S.F.: Existence and uniqueness results for some nonlinear boundary value problems. J. Math. Anal. Appl. 198, 35-48 (1996)

13. Ferracuti, L., Marcelli, C., Papalini, F.: Boundary value problems for highly nonlinear inclusions governed by non-surjective phi-Laplacians. Set-Valued Var. Anal. 19(1), 1-21 (2011)

14. Franco, D., O'Regan, D.: A new upper and lower solutions approach for second order problems with nonlinear boundary conditions. Arch. Inequal. Appl. 1, 413-419 (2003)

15. Garcia-Huidobro, M., Manásevich, R., Zanolin, F.: A Fredholm-like result for strongly nonlinear second order ODS's. J. Differ. Equ. 114, 132-167 (1994)

16. Garcia-Huidobro, M., Manásevich, R., Zanolin, F.: Strongly nonlinear second-order ODE's with rapidly growing terms. J. Math. Anal. Appl. 202, 1-26 (1996)

17. Krytsi, S.T., Matzakos, N., Papageorgiou, N.S.: Nonlinear boundary value problems for second order differential equations. Czechoslov. Math. J. 55, 545-579 (2005)

18. Kyritsi, S., Matzakos, N., Papageorgiou, N.S.: Periodic problems for strongly nonlinear second-order differential inclusions. J. Differ. Equ. 183, 279-302 (2002)

19. Manásevich, R., Mawhin, J.: Periodic solutions for nonlinear systems with p-Laplacian-like operators. J. Differ. Equ. 145 367-393 (1998)

20. Marcelli, C.: Existence of solutions to boundary-value problems governed by general non-autonomous nonlinear differential operators. Electron. J. Differ. Equ. 2012, 171 (2012)

21. Marcelli, C.: The role of boundary data on the solvability of some equations involving non-autonomous nonlinear differential operators. Bound. Value Probl. 2013, 252 (2013)

22. Marcelli, C., Papalini, F.: Boundary value problems for strongly nonlinear equations under a Wintner-Nagumo growth condition. Bound. Value Probl. 2017, 183 (2017)

23. O'Regan, D.: Existence theory for $\left(\phi\left(y^{\prime}\right)\right)^{\prime}=q f\left(t, y, y^{\prime}\right), 0<t<1$. Commun. Appl. Anal. 1, 33-52 (1997)

24. Papageorgiou, N.S., Papalini, F.: Pairs of positive solutions for the periodic scalar p-Laplacian. J. Fixed Point Theory 5 , 157-184 (2009)

\section{Submit your manuscript to a SpringerOpen ${ }^{\circ}$ journal and benefit from:}

- Convenient online submission

- Rigorous peer review

- Open access: articles freely available online

- High visibility within the field

- Retaining the copyright to your article

Submit your next manuscript at $\boldsymbol{~ s p r i n g e r o p e n . c o m ~}$ 